\title{
Diagnostic Value of Immunohistochemistry in Soft Tissue Tumors
}

\author{
Sridevi. V*., Susruthan Muralitharan., and Thanka. J \\ Dept of Pathology, SriMuthukumaran Medical college and Hospital, Chennai (India)
}

\section{ABSTRACT}

Background: Diagnosis of soft tissue tumors is a great challenge to pathologist but with the help of immunohistochemistry (IHC), proper analysis and diagnosis of soft tissue tumours can be made easy. The main use of immunohistochemistry in soft tissue neoplasms especially in sarcomas is to identify differentiation in the neoplastic cells.IHC are used as a panel or as single marker depending on the tumor.

Methods: A total of 513 soft tissue tumor (STT) cases were collected and reviewed. The cases were separated into as benign, intermediate and malignant cases. In 90 cases of STT in which immunohistochemistry (IHC) were used was further analysed and classified depending on the various positivity and negativity of the marker used.The significance of IHC was also analysed.

Result: In our study of 513 cases of STT there were 380 benign cases, 90 malignant cases and 43 intermediate cases. A total of 90 cases of sarcomas were present out of which 54\% cases required IHC, $20 \%$ cases required IHC to support the diagnosis but $26 \%$ of cases did not require IHC, the diagnosis was made on haematoxylin and eosin (H\&E). The IHC markers helped in correct diagnosis of STT cases.

Conclusion: Immunohistochemistry plays an important role in grading and giving precise diagnosis of soft tissue tumors. So it is important to use IHC to diagnose STT where haematoxylin and eosin did not give a precise diagnosis. Perfect diagnosis of STT helps in the correct therapeutic management of patients.

Keywords: Soft Tissue Tumors, Immunohistochemistry Markers, Soft Tissue Sarcomas, Diagnosis.

\section{Introduction}

Soft tissue tumours (STT) are composed of numerous different tumour types that are classifiedaccording to the type of mesenchymal tissue which they resemble. ${ }^{[1]}$ Immunohistochemistry (IHC) plays an important role in STT diagnosis. ${ }^{[2]}$ Immunohistochemistry has been introduced in the 80's and it is the application of immunologic principles to the study of cells and tissues. ${ }^{[3]}$ IHC helps to rule out a non-mesenchymal tumor, followed by trying to define mesenchymal cell lineage. As there are many complex diagnostic entities recognized of an intermediate malignant category including some tumors with a deceptively bland histological appearance, it is important to diagnose these tumours. ${ }^{[4,5,6]}$ The use of progressively small amounts of tissue for diagnosis highlights the importance of IHC. Immunohistochemistry will not be a substitute for skilled interpretation of conventionally stained microscopic specimens. As STT have several line of differentiation, numerous pseudo benign lesion and non mesenchymal malignant tumor are present which has to be differentiated for therapeutic reasons. So IHC is the major tool and plays an important role in determining the diagnosis of most of the soft tissue sarcomas. Panel of markers has to be used and quality of IHC technique and interpretation is also important. ${ }^{[2]}$ But wide "random" panels can be misleading.
Because of the lack of sensitivity or specificity of markers, and of frequent "aberrant" immunoreactivities, the use of a single immunostain can lead to misdiagnoses. It is difficult to recommend panels as the choice of antibodies will change according to the specific clinicopathological differential diagnosis. However, a few "basic" panels can be suggested, depending on the morphological category of the tumor.

\begin{tabular}{|l|l|}
\hline Histogenesis & Markers \\
\hline Mesenchymal(general) & Vimentin \\
\hline Epithelial & $\begin{array}{l}\text { Cytokeratins (CK), epithelial } \\
\text { membrane antigen (EMA) }\end{array}$ \\
\hline Smooth Muscle & $\begin{array}{l}\text { Desmin, smooth muscle actin } \\
\text { (SMA) }\end{array}$ \\
\hline Skeletal Muscle & Myoglobin \\
\hline Fibrohistiocytic & CD68 \\
\hline Endothelial & CD34,CD31 \\
\hline Lipomatous & $\begin{array}{l}\text { Immunohistochemistry } \\
\text { generally not used }\end{array}$ \\
\hline $\begin{array}{l}\text { Neuroendocrine, } \\
\text { Ewing's sarcoma/ PNET }\end{array}$ & $\begin{array}{l}\text { Neuron-specific enolase, } \\
\text { CD99 }\end{array}$ \\
\hline
\end{tabular}

"Aberrant positivities", such as the frequent expression of cytokeratins in leiomyosarcomas, Ewing sarcomas or 
epithelioidangiosarcomas should be known. ${ }^{[7]}$ The type of positivity whether nuclear, cytoplasmic, membranous and expected extent like diffuse or focal positivity should also be known. The extent of the immunoreactivity is also important e.g. S100 will be diffusely positive in benign nerve sheath tumors but only focally positive in MPNSTs.

As the treatment is different, it is extremely important to always rule out a non-sarcomatous tumor: carcinoma, melanoma, and lymphoma using adequate immunostains. Some of the commonly used markers are discussed below.

Epithelial markers Cytokeratins and EMA should be included in the immunohistochemical panel of most spindle and pleomorphic cell malignant tumors.

Lymphoid markers LCA, TdT should be included in the differential diagnosis of round cell neoplasms. Desmin is positive in rhabdomyosarcomas and in 70-90\% of smooth muscle tumors. Caldesmon it is relatively specific for smooth muscle differentiation. ${ }^{[8,9]}$ Myogenin is very specific in smooth muscle differentiation.

CD31is the most sensitive and specific vascular marker is, which is expressed by $95-100 \%$ of benign and malignant vascular tumors. However, the expression of CD31 by macrophages is a potential pitfall. CD68 is useful in the identification of histiocytic proliferations or reactive lesions. CD99 is expressed by most Ewing sarcomas, in a membranous fashion. FLI-1 expression in Ewing sarcoma / PNET is characteristic. Vimentin that is still widely used, although it does not provide any reliable diagnostic information. Vimentin is an important marker to detect the preservation of antigenicity of tissues. Ki 67 is the most commonly used proliferation marker. Ki-67staining is a more useful procedure than counting mitotic figures for assessing prognosis of the patients with sarcoma.

\section{Materials and Methods}

From January 2006 to June 2011 all soft tissue tumor diagnosed in the department of pathology, Sri Ramachandra
Medical College and ResearchInstitute were retrieved from the surgical pathology files and analysed. A total of 513 cases were collected and reviewed. Out of these cases benign, intermediate and malignant cases were identified. The cases which lacked details of important clinical and pathological features were excluded from the study. The gross characteristics of the tumor which included the tumor location, size, necrosis, circumscription, cut section and secondary changes were obtained from the pathology report registers. Hematoxylin and eosin (H\&E) stained sections of all the STT were reviewed and analysed. All the STT cases were analysed on the basis of cases which needed IHC for confirmation of the diagnosis, tumors which were diagnosed based only on $\mathrm{H} \& \mathrm{E}$ without IHC and cases which needed IHC for diagnosis. Most of the cases which needed IHC were malignant soft tissue tumor.

\section{Result}

Our study was a retrospective study in which we had analysed 513 cases of soft tissue tumours and retrieved cases which needed IHC in the period between January 2006 to June 2011, in the Department of Pathology, SRMC and RI.

There were 380 benign cases, 90 malignant cases and 43 intermediate cases. A total of 90 cases of sarcomas were present. $16 \%$ of synovial sarcomas were common among STT sarcomas followed by $14 \%$ fibrosarcoma and $13 \%$ leiomyosarcoma. (Fig: 1, Table: 1) Out of these, many STT cases needed IHC for diagnosing and confirmation. So the analysis of usefulness of IHC in sarcomas showed that it was not needed for the diagnosis of sarcomas in $26 \%$ of cases which was done on H\&E. IHC was done to support the haematoxylin and eosin diagnosis in $20 \%$ of case and diagnosing $54 \%$ of sarcoma cases was possible only with IHC.

\section{Discussion}

Soft tissue sarcomas (STSs) are heterogeneous group of malignant tumors with a wide spectrum in terms of

Table: 1: Total number of histological types of STT.

\begin{tabular}{|c|c|c|}
\hline S.NO & Histological type & Total cases \\
\hline 1. & Benign & 380 \\
\hline 2. & Intermediate & 43 \\
\hline 3. & Malignant & 90 \\
\hline & Total cases & 513 \\
\hline
\end{tabular}

Table 2: Frequency of various histological types of sarcomas.

\begin{tabular}{|l|c|c|}
\hline HISTOLOGICAL TYPE & FREQUENCY & PERCENTAGE \\
\hline Synovial Sarcoma & 16 & $16.5 \%$ \\
\hline Liposarcoma & 8 & $8.2 \%$ \\
\hline
\end{tabular}




\begin{tabular}{|l|c|c|}
\hline HISTOLOGICAL TYPE & FREQUENCY & PERCENTAGE \\
\hline Fibrosarcoma & 14 & $14.4 \%$ \\
\hline Leiomyosarcoma & 13 & $13.4 \%$ \\
\hline Rhabdomyosarcoma & 5 & $5.2 \%$ \\
\hline MPNST & 4 & $4.2 \%$ \\
\hline Ewing's/PNET & 5 & $5.2 \%$ \\
\hline Angiosarcoma & 1 & $1 \%$ \\
\hline Alveolar sarcoma & 1 & $1 \%$ \\
\hline DSRCT & 1 & $1 \%$ \\
\hline Pleomorphic sarcoma & 8 & $8.3 \%$ \\
\hline ExtraskeletalChondrosarcoma & 1 & $1.0 \%$ \\
\hline SclerosingEpitheloidFibrosarcoma & 2 & $2.1 \%$ \\
\hline Low Grade Fibromyxoid Sarcoma & 1 & $1 \%$ \\
\hline DFSP & 7 & $7.2 \%$ \\
\hline High Grade STS & 10 & $10.3 \%$ \\
\hline Total & $\mathbf{9 7}$ & $\mathbf{1 0 0} \%$ \\
\hline
\end{tabular}

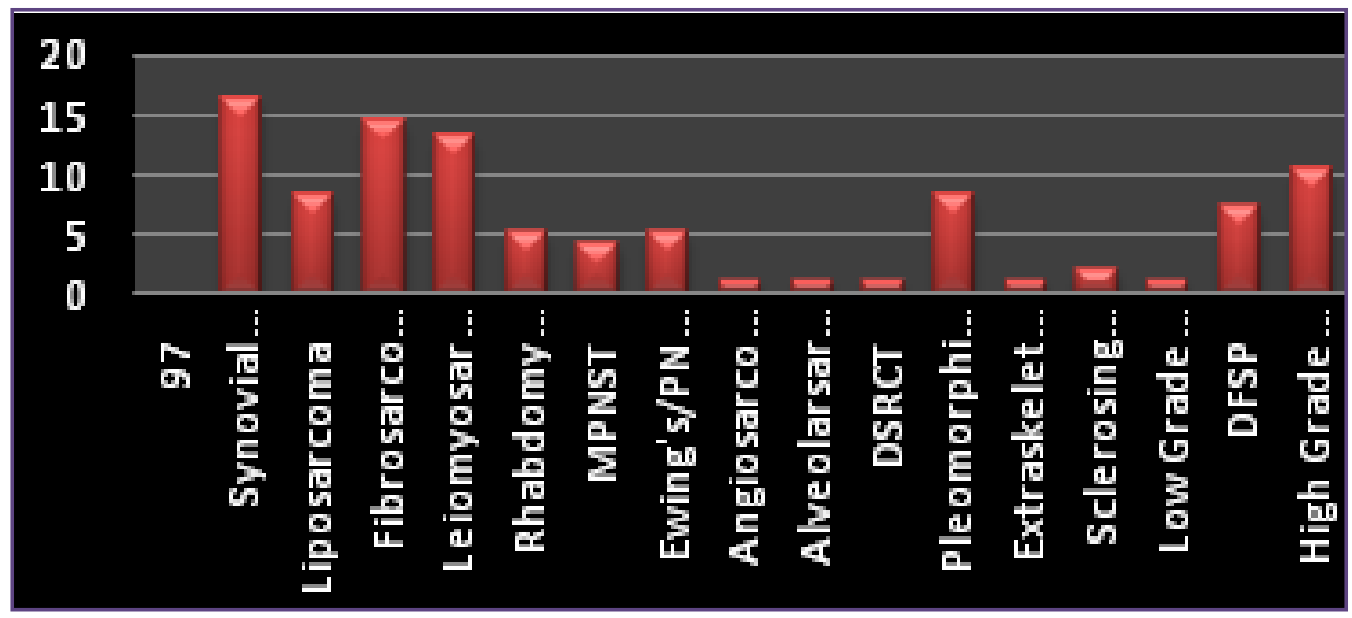

Fig. 1: Various histological subtypes of sarcomas.

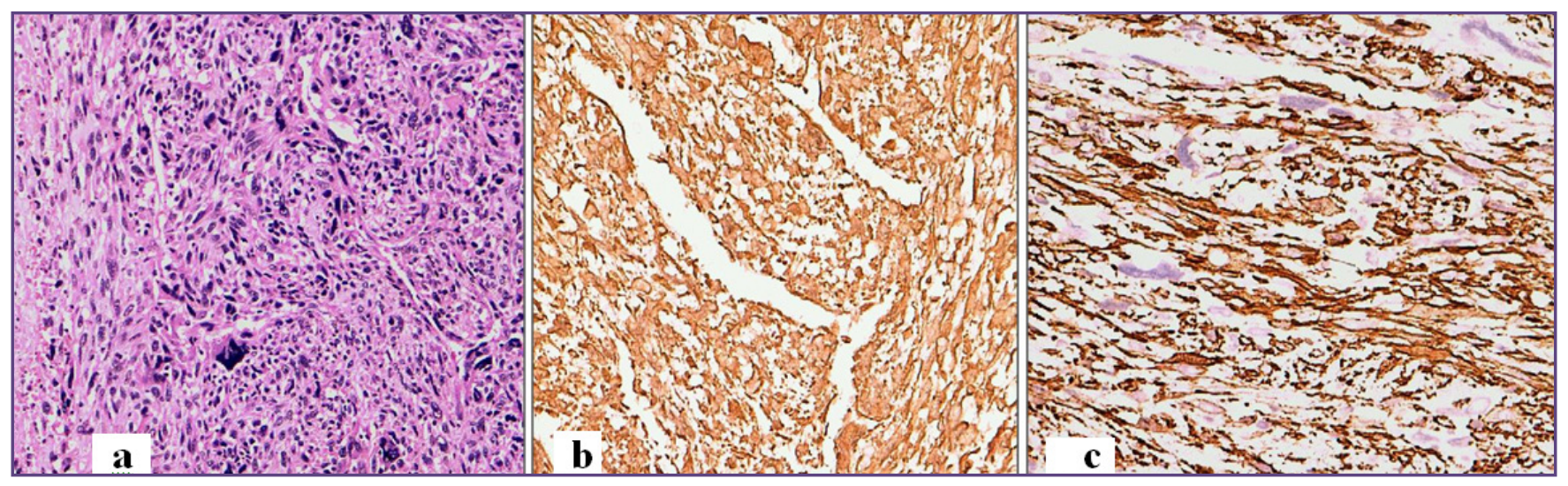

Fig. 2a: H\&E.10x-Pleomorphic Leiomyosarcoma: Highly pleomorphic cells with giant cells.; 2b: 20X - Immunohistochemical stains for Vimentin and 2c: SMA shows diffuse cytoplasmic positivity in the tumour cells. 


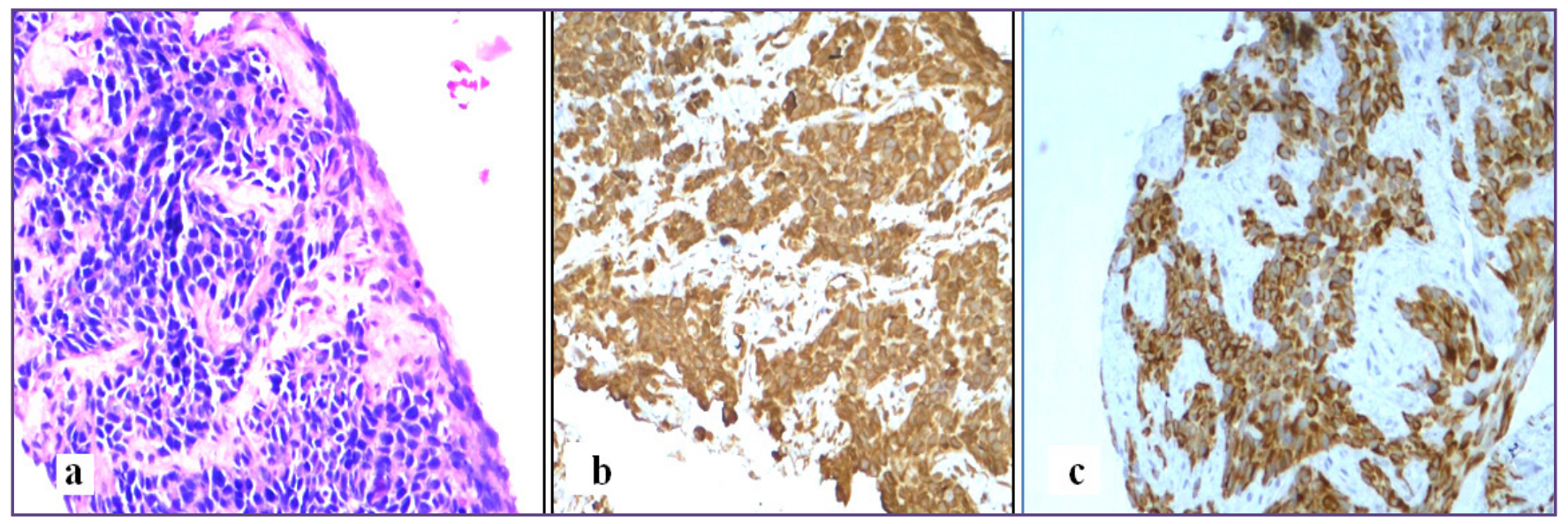

Fig. 3a: H\&E. 10x -Rhabdomyosarcoma :Small round blue cells with moderate amount of eosinophilic cytoplasm. 3b: 20x -Immunohistochemical stains for Vimentin and 3c :Desmin shows cytoplasmic positivity in the tumor cells.

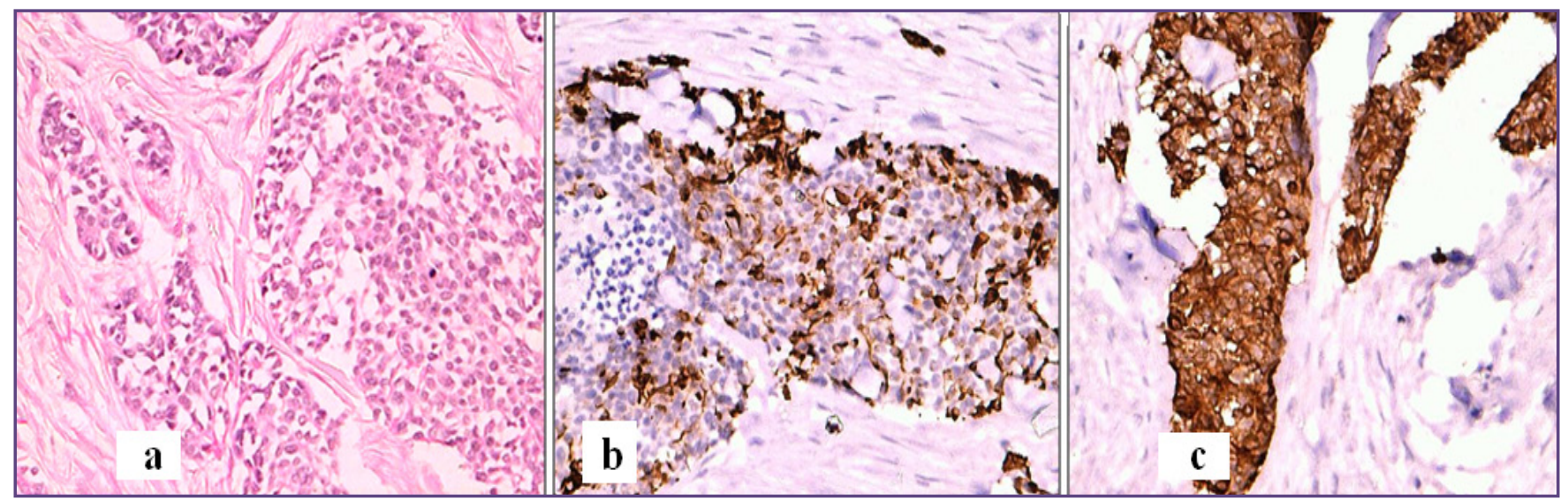

Fig. 4a: H\&E. 100x - DSCRT : Sheets of small round cells surrounded by desmoplastic stroma.4b: 100x :Desmin shows diffuse cytoplasmic. 4c: EMA membrane positivity in the tumour cells.

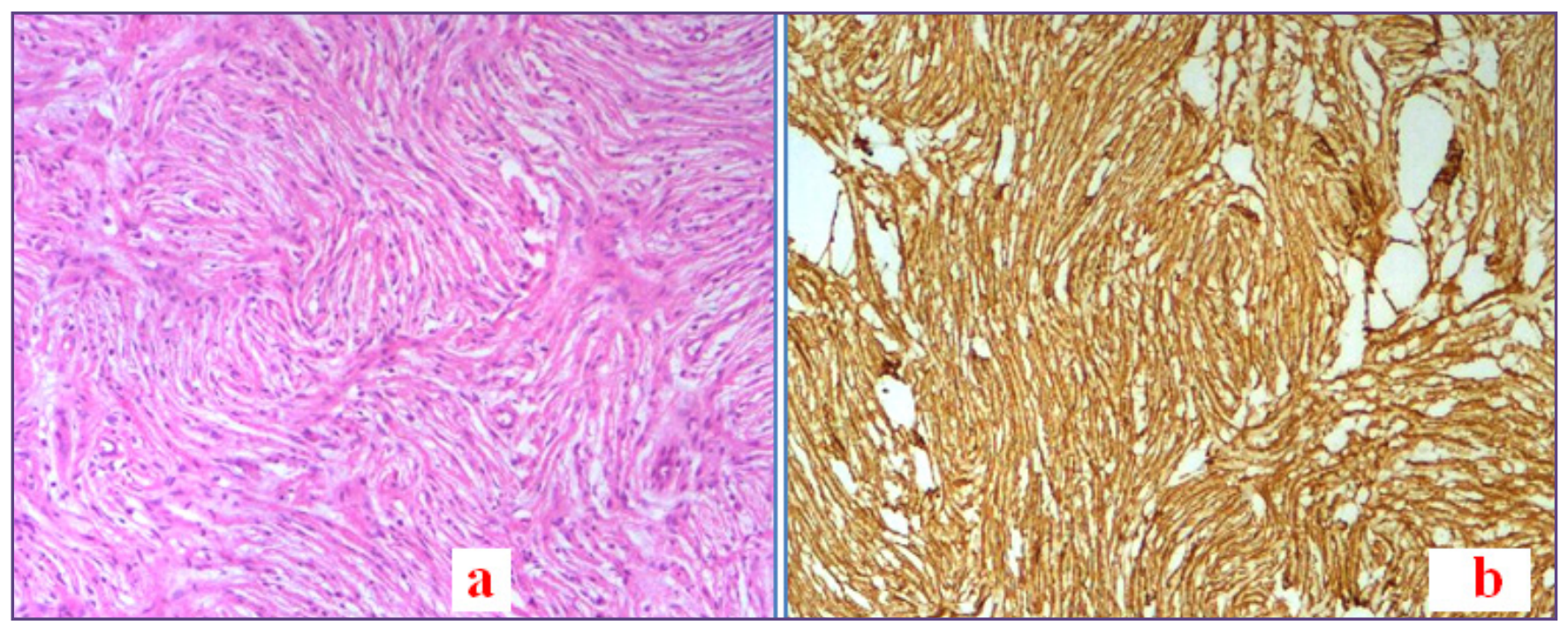

Fig. 5a: H\&E. 100X-DFSP :Spindle shaped cells arranged in storiform pattern. 5b:200x CD34 shows cytoplasmic and membrane positivity in the tumour cells. 
histologic type and prognosis. ${ }^{[1]}$ STT has been classified according to the WHO as benign, intermediate and malignant. It can occur in any age group. Histological grade of sarcomas is the most important prognostic factors. A total of 513 cases were studied, of which 380 cases were benign, 43 cases were intermediate and 90 cases were malignant. The study was undertaken to analyze the importance of immunohistochemistry (IHC) in soft tissue tumours. Among the immunohistochemical analysis of malignant tumours $54 \%$ of cases required immunohistochemistry (IHC) for the diagnosis, were as in $20 \%$ of cases IHC was useful to support the diagnosis. Immunohistochemistry is the most valuable adjuvant to hematoxylin and eosin staining in diagnostic histopathology. It is important in diagnosis of STT because of their variety with several lines of differentiation and the frequent difficulty of diagnosis with numerous psedosarcomatous benign lesion and non mesenchymal tumours. JM Coindre et al has shown that IHC is part of the definition of the tumours and is determinant for the diagnosis of rabdomyosarcoma (RMS), desmoplastic small round cell tumour (DSRCT) and synovial sarcoma.(Fig:2,3,4) Desmin and myogenin will be positive in the diagnosis of RMS. ${ }^{[10]}$ Our study also showed that these sarcomas needed IHC for the diagnosis. The desmin marker was useful in our study to identify all the RMS. Truong lo et al has also shown the same utility of desmin in RMS. As myogenin is more specific to skeletal muscle differentiation it is useful in diagnosing alveolar RMS. ${ }^{[1]}$ Malignant vascular tumours like angiosarcoma also need IHC to diagnose. CD 31 is the best marker for vascular lesion. In our study synovial sarcomas with biphasic pattern, IHC was useful to confirm the diagnosis. But in monophasic and poorly differentiated subtype IHC has to be done to diagnose the sarcomas. Folpe $\mathrm{Al}$ and Lopes JM et al studies showed that IHC is useful for the diagnosis of monophasic and poorly differentiated synovial sarcomas $^{[12,13]}$ which correlated with our study. For the synovial sarcoma cases for which Bcl-2 was needed, it was done and positive reaction was present. Suster $\mathrm{S}$ et al has also shown that the Bcl2 is positive in synovial sarcomas but it is a non specific marker ${ }^{[14]} \mathrm{A}$ DSRCT was present among the sarcomas in our study which showed desmin, CK and EMA positive with SMA positivity in the stroma. Ordonez NG et al IHC finding of DSRCT with 90\% positivity for CK, EMA and desmin with dot like positivity.

JM Coindre et al studies also showed that IHC may be helpful in the diagnosis of sarcomas like Ewing's sarcoma, Leiomyosarcoma, Dermatofibrosarcoma protuberance (DFSP) and myxoid liposarcoma (Fig:5) and it is also useful to support the diagnosis. For low grade sarcomas like DFSP IHC CD34 is positiveand it is done to rule out benign fibrous histiocytoma. ${ }^{[15]}$ The DFSP cases in our study showed storiform pattern of cell arrangement but CD 34 stain was done to support the diagnosis. Del Tos AP et al study showed the diagnostic utility of S100 in myxoid Liposarcoma which can be positive in some cases. Few of our myxoid liposarcoma (LPS) also showed S100 focal positivity.

Sarcomas with no specific immmunohistochemical profile is seen in fibrosarcoma, pleomorphic malignant fibrous histiocytoma (MFH), these sarcomas will not display any specific marker.JM Coinder study showed IHC may be useful to rule out other sarcomas with a specific line of differentiation. The nuclear proliferative antigen Ki67 is identified to correlate with the prognosis of the patients to predict the outcome and distant metastasis of sarcoma patients. A significant correlation has been reported between Ki67 expression and mitotic rate in sarcomas. ${ }^{[16]}$ A correlation between ki67 reactivity and tumour grade in sarcomas has been detected in different retrospective studies. The findings in our study indicate that precise use of immunohistochemical stain can be a valuable adjunct to routine histopathology, exact grading of the sarcomas and in treatment management of patients.

\section{Conclusion}

Our study concludes that immunohistochemistry is an important tool in giving precise diagnosis of soft tissue tumours and it should be done as a panel to diagnose cell of origin.IHC can be done on routine basis to diagnose undifferentiated STT, confirm the diagnosis and to accurately grade the sarcomas so that it will be beneficial for the management of the patient and preoperative and postoperative treatment planning would be possible.

\section{Reference}

1. Fisher C. Immunohistochemistry in Diagnosis of Soft Tissue Tumours. Histopathology. Wiley, 2010; 58 (7):1001.

2. Heim-Hall J, Yohe SL. Immunohistochemistry of Soft Tissue Neoplasms. ArchPathol Lab Med. 2008; 132:476-489.

3. Coindre JM. Immunohistochemistry in the diagnosis of soft tissue tumours. Histopathology. 2003; 43: 1-16.

4. Fisher C. The comparative roles of electron microscopy and immunohistochemistry in the diagnosis of soft tissue tumours. Histopathology. 2006;48:32-41.

5. Hornick JL. Novel uses of immunohistochemistry in the diagnosis and classification of soft tissue tumors. Modern Pathology. 2014; 27: 47-63.

6. Suster S. Recent advances in the application of Immunohistochemical markers for the diagnosis of soft tissue tumours. Semin. Diagn. Pathol. 2000; 17: 225-235. 
7. de Saint N, Somerhausen A. Immunohistochemistry in the diagnosis of soft tissue tumors. Path 2005: 2-12.

8. Ceballos KM, Nielsen GP, Selig MK, O' Connell JX. Is antih-caldesmon useful for distinguishing smooth muscle and myofibroblastic tumors? An immunohistochemical study. Am. J. Clin. Pathol. 2000; 114: 746-753.

9. Yves-Marie Robin etal. Transgelin is a novel marker of smooth muscle differentiation that improves diagnostic accuracy of leiomyosarcomas: a comparative immunohistochemical reappraisal of myogenic markers in 900 soft tissue tumors. Modern Pathology.2013; 26: 502-510

10. Kumar S, Perlman E, Harris CA et al. Myogenin is a specific marker for rhabdomyosarcoma: an immunohistochemical study in paraffin- embedded tissues. Mod. Pathol. 2000; 13: 988-993.

11. Dias P Chen B, Dilday B et al. Strong immunostaining for myogenin in rhabdomyosarcoma is significantly associated with tumors of the alveolar subclass. Am. J. Pathol. 2000; 156: 399-408.
12. Olsen SH, Thomas DG, Lucas DR. Cluster analysis of immunohistochemical profiles in synovial sarcoma, malignant peripheral nerve sheath tumor, and Ewing sarcoma. Mod Pathol. 2006;19:659-668.

13. YangLetal.Clinical pathologicalanalysis of synovial sarcoma. Chinese Journal of clinical oncology.2007;4:246-249.

14. Cunha KS et al. Evaluation of Bcl-2, Bcl-x and Cleaved Caspase-3 in Malignant Peripheral Nerve Sheath Tumors and Neurofibromas .Annals of the Brazilian Academy of Sciences. 2013 March.

15. Li N, McNiff J, Hui $P$, et al. Differential expression of HMGA1 and HMGA2 in dermatofibroma and dermatofibrosarcomaprotuberans: potential diagnostic applications, and comparison with histologic findings, CD34, and factor XIIIa immunoreactivity. Am J Dermatopathol.2004;26:267-272.

16. Gupta, et al., Typing and Grading of Soft Tissue Tumors and their Correlation with Proliferative Marker Ki-67. J CytolHistol. 2015; 6:3.

*Corresponding author:

Dr Sridevi. V, 52(Old No:39), Gangadeeshwaran Koil Street, Nutech-Sreenivas Apartment, Flat:C-9, Purasaiwalkam, Chennai (India):600084

Phone: +91 09444952854

Email: drsridevi78@yahoo.co.in

Date of Submission : 06.08.2017

Date of Acceptance : 30.08.2017

Financial or other Competing Interests: None.

Date of Publication : 22.12.2017 